\title{
Os efeitos do mindfulness na percepção dos estudantes de medicina de uma universidade brasileira
}

\section{The effects of mindfulness as perceived by Brazilian medicine students}

\author{
Laura Leísa Silveira' (D) |lauraleisa@gmail.com \\ Heron Eduardo Fabrizzi' (1) heronfabrizzi@gmail.com \\ Henrique Cesar Correa Hamilko' (D) henriquehamilko@gmail.com \\ Sabrina Stefanello' (D) binastefanello@gmail.com \\ Deivisson Vianna Dantas dos Santos' (D) deivianna@gmail.com
}

\section{RESUMO}

Introdução: Sob a perspectiva do cuidado em saúde do estudante, o bem-estar mental desempenha forte influência na qualidade de vida e, consequentemente, na performance acadêmica e profissional. Programas baseados na atenção plena focam o desenvolvimento de habilidades de gerenciamento de estresse, sendo estratégias eficazes para melhorar o manejo da saúde mental. Ademais, esses programas se mostram reproduzíveis e com a característica de serem realizados em grupo.

Objetivo: Este estudo teve como objetivo analisar a percepção sobre um programa de prática meditativa oferecido a estudantes de Medicina com o enfoque nas estratégias apreendidas a partir dessa experiência.

Método: Este estudo foi realizado na Universidade Federal do Paraná, por meio da aplicação de um protocolo preexistente de mindfulness adaptado para a realidade dos estudantes de graduação. Trata-se de um estudo de metodologia qualitativa que avaliou os impactos trazidos pela meditação e as implicações efetivas na vida dos participantes do programa.

Resultados: Significativas mudanças foram relatadas pelos participantes, como um autocuidado maior, melhora na organização pessoal e maior compreensão de suas emoções. Apontaram a estratégia como ponto de apoio importante para enfrentamento das dificuldades diárias que encontravam no curso. Tais aspectos perduraram nos discursos dos participantes mesmo após seis meses da intervenção. Apesar dessa melhora, os alunos não incorporaram a meditação em si como um hábito diário.

Conclusões: Na perspectiva de uma abordagem em grupo, além dos efeitos positivos, a prática se mostrou barata e de fácil reprodução. Por se tratar de uma prática com diversos protocolos estabelecidos e bem estudados, poderia se tornar uma inestimável ferramenta de apoio à saúde mental dos estudantes.

Palavras-chave: Atenção Plena; Saúde Mental; Estudantes de Medicina.

\section{ABSTRACT}

Introduction: From the perspective of student health care, mental well-being is a key influence on quality of life and, consequently, on academic and professional performance. Mindfulness-based programs focus on developing stress management skills, representing effective strategies to improve mental health management. Furthermore, these programs are reproducible and can be conducted in groups.

Objective: To analyze how medical students perceive a practical meditation program offered to them, focusing on the strategies learned from that experience.

Method: This study was conducted at the Federal University of Paraná, using an existing Mindfulness protocol adapted to the reality of the undergraduate students. It was a qualitative methodological study to assess the impacts caused by the meditation and the effective life implications for the participants.

Results: Significant changes were reported by the participants, such as greater self-care, improved personal organization and greater understanding of their emotions. They pointed out the strategy as an important support point to face the daily difficulties they encountered during medical school. Such aspects persisted in the accounts given by the participants even after six months of the intervention. Despite this improvement, the students did not incorporate meditation itself as a daily habit.

Conclusions: From a group approach perspective, as well as the positive effects, the practice proved to be cheap and easy to reproduce. As there are numerous well-studied and established protocols for the practice, it could become an invaluable support tool for student mental health.

Keywords: Mindfulness; Mental Health; Medical Students.

${ }^{1}$ Universidade Federal do Paraná, Curitiba, Paraná, Brasil.

Editora-chefe: Daniela Chiesa

Editor associado: Maurício Abreu Pinto Peixoto

Recebido em 14/05/20; Aceito em 05/02/21.

Avaliado pelo processo de double blind review. 


\section{INTRODUÇÃO}

Estudos sugerem que estudantes de Medicina têm maiores índices de estresse ${ }^{1}$ e suicídio ${ }^{2}$, e maior prevalência de desordens mentais quando comparados à população em geral $^{3}$. Esses problemas de saúde mental são normalmente relacionados à piora no rendimento acadêmico ${ }^{1,4}$, à menor empatia com os outros e à tendência ao isolamento ${ }^{5,6}$, tendo implicações na qualidade de vida desses estudantes.

Essa é uma questão que merece ser valorizada em um contexto educacional, e medidas que vão desde o suporte ao desenvolvimento pessoal até projetos pedagógicos centrados nos estudantes deveriam ser consideradas. A educação médica não deve separar aspectos que envolvam o desenvolvimento acadêmico da saúde mental? .

Fatores comportamentais como rigidez emocional, tentativa de controle excessivo de emoções, projeções fantasiosas sobre o futuro e comportamento compulsivo parecem ser mais importantes do que fatores ocupacionais para a gênese de sofrimentos mentais experimentados por essa população ${ }^{8}$. Dyrbye et al. ${ }^{9}$ mostraram que uma das possíveis soluções para esse problema seria a criação de programas que ensinassem aos estudantes habilidades para controle de estresse e aumento da consciência sobre seus estados emocionais. Intervenções com meditação, especialmente aquelas baseadas em protocolos mindfulness, podem prover essas e muitas outras habilidades, como aumento da motivação, modulação da atenção e sociabilidade ${ }^{10}$. A meditação como uma terapia cognitiva surgiu da crença de que a observação de pensamentos, sentimentos e sensações corporais poderia ajudar as pessoas a conhecer as suas próprias preocupações mentais, adquirindo a capacidade de controlar seus processos mentais; além disso, essa prática é altamente econômica ${ }^{11}$.

No Brasil, as práticas meditativas foram aprovadas como intervenção válida pelo Sistema Único de Saúde somente em 2017 ${ }^{12}$. Mindfulness é um termo ainda pouco familiar tanto para a população geral quanto para a comunidade acadêmica. Apesar de ainda pouco difundida, essa ferramenta já é uma estratégia de intervenção em vários setores sociais, incluindo o acadêmico ${ }^{13}$.

A prática regular e contínua de meditação pode oferecer ao indivíduo um novo entendimento de conceitos sobre si, o mundo, a sociedade e a natureza da experiência ${ }^{14}$. A prática da meditação tem sido usada como estratégia de intervenção em vários setores da sociedade, incluindo o estressante ambiente acadêmico $^{13}$. Na prática clínica, o uso dessas terapias já se mostrou benéfico para uma série de condições, como depressão, ansiedade ${ }^{15}$, abuso de drogas ${ }^{16}$, transtornos alimentares ${ }^{17}$, insônia ${ }^{18}$, dor crônica $^{19}{ }^{\text {, }}$ câncer $^{20}$, psoríase ${ }^{21}$, fibromialgia, artrite reumatoide e déficits de atenção e hiperatividade ${ }^{22}$. Apesar do crescente interesse em sua aplicação e efeitos, alguns autores enfatizam que esse tipo de protocolo é ainda alvo de interpretações equivocadas, principalmente quanto ao seu conceito e sobre como opera resultados ${ }^{23}$.

Entre as habilidades desenvolvidas por meio da meditação, chamamos a atenção para uma maior empatia ${ }^{24} \mathrm{e}$ a melhora da memória e da compreensão de leitura ${ }^{25}$, o que parece ser útil para melhorar o rendimento acadêmico. Por dialogarem simultaneamente com as causas e as consequências dos problemas comumente relacionados à saúde mental de estudantes, as intervenções baseadas em mindfulness podem ser uma ferramenta muito útil a essa população ${ }^{26,27}$. Ainda mais, sob a perspectiva da formação médica, qualidades como o baixo custo e a logística simples de implementação sugerem a potencial eficiência dessas intervenções como rede de apoio ao corpo discente universitário.

O alvo do estudo é, portanto, aprimorar o entendimento acerca de mecanismos e percepções surgidos a partir da prática meditativa proposta a um grupo de estudantes de Medicina. Ademais, investigamos a significação atribuída à experiência em grupo como possível rede de apoio dentro da universidade com o objetivo de compreender quais são as formas de oferecer suporte a esses acadêmicos para então melhorar essa rede.

\section{MÉTODOS}

Este estudo pode ser classificado como uma pesquisaação, estabelecida por Tripp ${ }^{28}$ como meio para descrever mudanças propostas a uma determinada abordagem, a fim de melhorar a prática vigente. Apesar do cunho pragmático, a pesquisa-ação difere da aplicação prática do recurso estudado, bem como da pesquisa científica tradicional. Isso se dá principalmente porque a pesquisa-ação tanto identifica as estratégias para aperfeiçoar um sistema existente quanto submete o que se entendeu como aprimoramento de reflexões sistemáticas, de modo a ressignificar o objeto de estudo ${ }^{28}$.

Ademais, trata-se de uma pesquisa qualitativa em saúde, uma vez que a observação dos sujeitos prioriza a profundidade sobre a quantidade, tendendo a fornecer maior entendimento sobre a questão estudada. Ao longo da pesquisa, a natureza multidimensional do fenômeno em sua manifestação natural esclarece o entendimento sobre o indivíduo, bem como sobre a influência da intervenção nesse contexto particular ${ }^{29}$.

Todos os procedimentos realizados neste estudo estavam em conformidade com os padrões éticos, sendo aprovados pelo Comitê de Ética em pesquisa local Certificado de Apresentação para Apreciação Ética (CAAE) no 64457317.3.0000.0102. O consentimento informado foi obtido de todos os participantes incluídos no estudo. 


\section{Intervenção}

A intervenção estudada foi adaptada do programa do livro Mindfulness-based cognitive therapy for depression ${ }^{30}$, que tem como base o programa Kabat-Zinn, e realizada em grupo. Apesar de existirem adaptações, manteve-se a estrutura original das sessões. Os áudios originais indicados para serem ouvidos durante as sessões práticas foram substituídos pela leitura das diretrizes traduzidas do livro para as meditações guiadas em grupo.

O programa durou dois meses e foi composto por sessões presenciais semanais de duas horas de duração. Além disso, guias para a realização das práticas diárias individuais foram fornecidos pelos pesquisadores. Tais práticas abrangiam compreensão corporal e percepção dos estados da mente durante o dia a dia. A meditação sentada com atenção à respiração, às sensações corporais e aos estados emocionais foi usada como exercício para habilitar a percepção corporal, e adotaram-se também as poses de ioga para trabalhar a consciência física. Nas sessões em grupo, havia rodadas de perguntas-respostas e reflexões sobre as experiências individuais da semana.

O recrutamento inicial foi feito por meio de anúncios em redes sociais e de avisos durante as aulas do curso. As sessões ocorreram dentro da estrutura da universidade, em sala de aula adaptada para esse propósito, onde os alunos se sentavam no chão com almofadas que traziam de casa, arranjados em círculo. Do total de 41 participantes que iniciaram a intervenção, dez compareceram em menos de duas sessões, dez em menos de três sessões e 21 compareceram a um mínimo de cinco sessões.

\section{Coleta e análise dos dados}

Os dados foram colhidos pelos próprios pesquisadores. Realizaram-se grupos focais em diversos momentos da vivência dos estudantes na prática de meditação: grupos focais pré-intervenção $(G 1, G 2)$, grupos focais logo após a intervenção $(G 3, G 4, G 5)$ e um último grupo focal realizado seis meses após a intervenção (G6). Os grupos focais préintervenção contaram com a participação de 41 pessoas. Os grupos pós-intervenção tiveram 21 participantes, enquanto, no grupo focal após seis meses da intervenção, compareceram nove pessoas. Um roteiro único para cada momento de realização dos grupos focais foi elaborado.

Grupos focais consistem em um modo de obter dados a partir de sessões em grupo em que as pessoas partilham as experiências comuns. Tais grupos permitem a coleta de informações relevantes sobre um assunto particular, o que possibilita a apreensão não só do que pensam os participantes, mas também dos porquês desses pensamentos. Além disso, é possível observar a interação entre os participantes do grupo e os diferentes graus de consenso e discordância ${ }^{31,32}$. Essa técnica é amplamente utilizada nas áreas de saúde, educação e sociologia para a coleta de dados e avaliação de programas e serviços ${ }^{33}$.

Os grupos focais foram gravados e transcritos. Preservouse a transcrição dos trechos, e aqueles similares em sua temática foram aglutinados em categorias semânticas a partir de uma leitura hermenêutica do material ${ }^{34,35}$. A hermenêutica é entendida não como uma metodologia em si, mas como uma postura interpretativa que se sustenta pela busca da compreensão de um texto ou de um fenômeno humano, posicionando-se entre a explicação da natureza e a compreensão da história ${ }^{35}$. Visa, portanto, compreender de modo interpretativo os processos de construção de sentido e o universo de significações situados em determinado momento histórico.

Para seguir a dinâmica hermenêutica, foi feito um processo de leituras inicialmente superficial das transcrições, e, após, iniciou-se o processo de transformar os textos em narrativas, em que se manteve o sentido das falas originalmente criadas pelos participantes. Buscou-se, também, sintetizar os textos, enfatizando ideias repetidas. Apresentaram-se exemplos de forma resumida, e os vícios de linguagem foram transformados em escrita, para tornar o texto mais corrido e compreensível, sem perder a essência das falas dos participantes. Segue-se assim o preceito hermenêutico de buscar o significado a partir dos fenômenos emanados dos discursos, extraindo-se categorias semânticas sem realizar interpretações, a priori, do material ${ }^{35}$.

Avaliamos, em seguida, as diferenças e semelhanças entre os textos das narrativas dos diferentes grupos. Procuramos entender as explicações para as diferentes experiências, que impactos a vivência teve no curso de Medicina e as possíveis mudanças provocadas pela prática da atenção plena.

As categorias semânticas estão listadas no Quadro 1, de modo que as ideias centrais foram comparadas entre si. Após a primeira análise das transcrições para a construção das categorias, uma segunda análise foi realizada por um pesquisador diferente, com o intuito de reduzir o viés interpretativo. Com relação aos dados, adotou-se a sequência demonstrada na Figura 1 e analisou-se um total de oito horas e meia dos sete grupos focais realizados.

\section{RESULTADOS E DISCUSSÃO}

Todos os participantes eram estudantes do curso de Medicina da Universidade Federal do Paraná. Três grupos focais iniciais foram realizados com 12, 16 e 13 participantes cada. Dos 41 participantes que iniciaram o programa de meditação, 21 estudantes compareceram a um mínimo de cinco sessões, dez participaram de um mínimo de três reuniões e dez desistiram do programa, tendo participado de menos de duas sessões. No 
final da intervenção, os 21 alunos com maior frequência foram separados em dois grupos focais com nove e doze pessoas, dos quais 15 eram mulheres e seis homens, com idades entre 17 e 26 anos. O primeiro, quinto e nono períodos letivos do respectivo curso não tiveram participantes nos grupos finais. A distribuição entre os demais períodos $\left(2^{\circ}, 3^{\circ}, 4^{\circ}, 6^{\circ}, 7^{\circ}, 8^{\circ}\right.$, $10^{\circ}, 11^{\circ}$ e $12^{\circ}$ ) foi relativamente uniforme. Nove participantes participaram do grupo focal final seis meses após a intervenção.
A curiosidade sobre o método foi uma motivação recorrente para a participação na pesquisa. Além dela, muitos participantes referiram que os relatos de amigos ou familiares praticantes de meditação os influenciaram a experimentar a intervenção. Outras motivações incluíram experiências anteriores com técnicas meditativas e informações sobre as vantagens da prática. O Quadro 1 resume os principais achados que serão descritos a seguir.

Figura 1. Sequência da construção dos dados

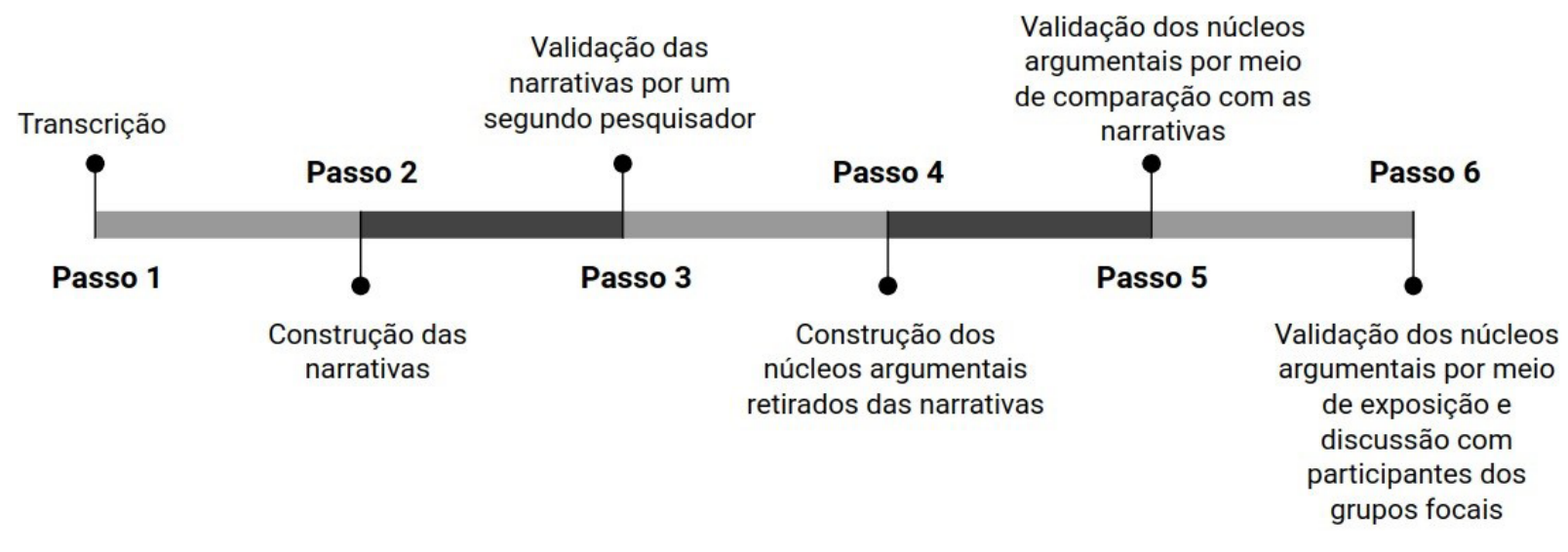

Quadro 1. Resumo dos principais achados qualitativos

\section{Categorias}

Motivações para iniciar o programa

Estratégias para lidar com o sofrimento

\section{A experiência relacionada à técnica}

Os estudantes relataram, nos grupos pós-intervenção, que vivenciaram um estado mais consciente e desenvolveram um autocuidado maior, exemplificado no sentido de alcançar a calma, diária

\section{Percepções apreendidas a partir das narrativas}

Oportunidade para conhecer a si mesmo e recuperar o senso de identidade. Curiosidade sobre a técnica.

Referências positivas de pessoas próximas sobre meditação.

Experiências anteriores com práticas meditativas.

Melhora da percepção pessoal em relação à sua saúde mental.

Possibilidade de ter na rotina um momento de autocuidado.

Melhora no autocuidado, na concentração e na autonomia emocional. Compartilhamento do sofrimento com outros componentes do grupo.

Maior consciência sobre si e sobre o ambiente que o cerca.

Aumento da capacidade de pequenas vivências do seu cotidiano.

Maior gentileza em detrimento de sentimento de culpa, conduzindo a estados mentais de menor remorso.

Consciência sobre os ciclos de pensamentos ruminativos e seus danos.

Exercício de mudança do foco da atenção como postura ativa diante da ruminação.

Maior entendimento sobre os próprios processos mentais, trazendo foco para lidar com estados indesejados.

Os desafios para incorporação da prática A alta demanda acadêmica na rotina dos participantes dificultou a conciliação melhorar a organização pessoal, aumentar a concentração e ter um maior controle de suas emoções. Os resultados encontrados estão de acordo com os obtidos por outros pesquisadores ${ }^{36-40}$. Segundo estas pesquisas, alguns dos temas que pareciam ser 
os principais fatores responsáveis pelas mudanças positivas nos participantes desse tipo de programa incluem a capacidade de retomar o autocontrole por meio da compreensão, conscientização e aceitação, e pelo impacto do grupo.

Na última [reunião] que eu vim eu percebi que eu tava mais capaz de [me] controlar e que isso acontecia no dia a dia também. Numa semana em que tive uma prova, consegui notar o estresse de todo mundo ao meu redor. Melhorei meu entendimento sobre as coisas ao meu redor. Quando você toma um remédio por causa do estresse de uma prova, por exemplo, você fica inerte, mas com a meditação você não fica assim, você percebe os motivos que te levaram ao estresse (G3).

Os estudantes relataram também que o aumento da autoconsciência ajudou no autocontrole, criando um tipo de "radar" para estados mentais considerados por eles mesmos como indesejados. Eles identificaram que a "ruminação de pensamentos" foi um dos processos mais danosos que vivenciavam e que foi identificado pela prática da meditação. $A$ ruminação é entendida como um foco passivo e repetitivo nos motivos e sintomas do estresse em si e em suas implicações, sem que o estudante tome uma postura ativa para trabalhar a questão geradora do mal-estar ${ }^{41}$. Rodrigues et al. ${ }^{37}$, em revisão de intervenções similares, identificaram a estratégia da atenção plena como clarificadora de processos de ruminação, assim como relatado em nosso estudo: "Acho que o que me ajuda é essa gentileza direcionada ao que eu sinto e sobre as coisas que eu não posso mudar e que tenho que aceitar como são" (G4).

Os estudantes notaram que, durante as conversas após as meditações, os participantes experimentaram um sentimento de que seu sofrimento não é exclusivo, mas sim compartilhado por outros membros do grupo. Esse tipo de identificação é chamado de universalização por alguns estudos ${ }^{42}$. Um tipo de sofrimento comum aos participantes, por exemplo, era a autocrítica e a cobrança excessiva em relação ao rendimento acadêmico. Ao mesmo tempo, essa troca entre os participantes da intervenção gerava uma coesão e um sentimento de pertencimento em relação ao grupo.

Eu realmente gostei de poder parar um dia na semana e ver que fazia parte de algo, o que é difícil. Com a [intervenção de] meditação eu vi pessoas de outros períodos passando por muitos problemas e saber com o que eu vou ter de lidar no futuro foi muito bom para $\operatorname{mim}(G 6)$.

Foi muito bom também para mim vir e poder ouvir os outros. Mesmo que a gente não fale com todo mundo que veio, todos começaram a ver os outros de um jeito diferente. Eu me senti mais conectada com pessoas que eu mal conhecia antes (G4).

Yalom et al. ${ }^{42}$ definem a coesão como elemento central para a conexão entre os membros de um grupo e a colocam como um fator tão importante para essa modalidade terapêutica quanto a relação entre terapeuta e cliente em terapias individuais. Em sua pesquisa, aparece como o terceiro ponto mais importante para o sucesso terapêutico em grupo.

\section{Melhorando as estratégias para lidar com o sofrimento}

Necessidades recorrentes relatadas pelos estudantes como de se permitirem sentir os próprios estados mentais sem a sensação de medo e de lutar contra o autobloqueio (que é uma tentativa de proteção contra pensamentos negativos) foram recorrentes nos grupos focais iniciais. A expectativa de melhorar o autoconhecimento foi também um destaque, com relatos trazendo a vontade de conhecer melhor os próprios processos cognitivos e de recuperar-se de uma perda de identidade ocorrida durante a graduação.

Os fatores que motivaram os estudantes a seguir frequentando as sessões, de acordo com falas dos grupos focais finais, incluíram: melhora do estado mental geral, desejo de avançar no conhecimento da técnica, compromisso com a decisão pessoal de seguir no programa e prazer de reservar um momento da semana para autocuidado.

A meditação me deu ferramentas para buscar um estado mental mais agradável. Os exercícios de observar os pensamentos passando como vídeos em uma tela branca, os de foco na respiração e no momento atual, foram presentes da prática pra mim (G4).

O nível de autoconhecimento que [a prática de mindfulness] gera me surpreendeu muito. Só o fato de você perceber [o que te deixa mal] já é uma grande vitória, porque se você tentar mudar algo que nem sabe o que é... não vai dar certo (G5).

Como nos estudos revisados por Cairns et al. ${ }^{38} \mathrm{e}$ no trabalho desenvolvido por Hjeltnes et al. ${ }^{43}$, pode-se perceber que os exercícios propostos pela intervenção se tornaram meios pelos quais os participantes lidam com pensamentos negativos. Além de a intervenção propor uma nova maneira de se relacionar com emoções negativas, para alguns participantes os elementos incorporados na rotina trouxeram benefícios inesperados como redução de sintomas quando se tratava de estudantes que já faziam seguimento por questões de saúde mental.

Além disso, puderam-se observar mudanças nos sentimentos que os participantes direcionaram a si mesmos, mesmo depois de seis meses após o término da intervenção. Em todos os grupos focais feitos após o término do programa, houve destaque sobre o quanto diminuíram os sentimentos de culpa, ao passo que aumentaram os de gentileza para consigo. Foram recorrentes, também, as falas de como essa transposição de sentimentos direcionados a si ajudou os participantes a 
diminuir o remorso ante momentos julgados como ociosos por eles mesmos, enfraquecendo o ciclo de fortalecimento de culpa que muitos vivenciavam: "Aceitar que tudo o que acontece faz parte me fez muito bem. Aprender a ser mais gentil comigo mesma mudou muito a minha percepção das coisas" (G6).

As percepções trazidas pelos estudantes de Medicina que participaram de nossa intervenção sugerem efeitos benéficos sustentados ao menos seis meses após o fim do programa de meditação aplicado. Para afirmar que as mudanças se estendem por períodos maiores, novos estudos, com acompanhamento mais longo, seriam necessários. Como os resultados de nosso estudo são consistentes com os de outros estudos, podemos analisar os dados disponíveis para adquirir mais conhecimento sobre a duração definida dos efeitos observados. Van Aalderen et al. ${ }^{44}$, por exemplo, mediram efeitos de uma terapia baseada em mindfulness por até 15 meses após a intervenção. Os testes mediram os sintomas depressivos, o nível de ruminação mental e as habilidades aprendidas durante o programa. Os dados obtidos três meses após a terapia mantiveram-se estáveis durante todo o seguimento, a não ser pelo nível de ruminação, que continuou em declínio durante todo o estudo. Munshi et al. ${ }^{45}$ observaram que, para os pacientes que mostraram remissão de sintomas depressivos após intervenção mindfulness, os efeitos duraram até cinco anos. De Vibe et al. ${ }^{46}$ demonstraram que os efeitos positivos sobre a saúde mental de estudantes de Medicina e de Enfermagem perduraram por seis anos, mesmo com a baixa aderência às práticas sugeridas no dia a dia.

\section{Os desafios para incorporação da prática diária de meditação}

A expectativa de incorporar o hábito da meditação como prática diária não foi alcançada pela maioria dos estudantes após seis meses da intervenção. Os estudantes associaram sua falta de autocuidado às exigências que o curso de Medicina impõe, cujas demandas os levam a um senso de perda de autonomia. Tais demandas foram exemplificadas pela carga horária alta, pela pressão pela participação em atividades extracurriculares, pelo número excessivo de disciplinas e por dificuldades com os desafios de aprendizado, o que resultava em autocobrança disfuncional. Críticas foram feitas sobre a incompatibilidade de se seguir com todas as atividades acadêmicas ao mesmo tempo que se lida com questões pessoais, como a morte de um familiar.

Apesar de a maioria dos estudantes não ter incorporado o hábito de meditar em sua rotina sob a justificativa da falta de tempo para tal, exercícios ensinados durante o programa foram em algum grau incorporados. Esse foi o caso para as práticas que envolviam o mindfulness ao comer, ao caminhar e de um exercício específico ensinado, denominado de "A prática dos três minutos". Esse exercício consistia em meditação de muito curto tempo utilizado para aumentar a consciência sobre si e sobre o momento presente. Os estudantes deram relatos muito positivos sobre como melhoraram o uso de seu tempo durante o dia, saindo de um modo passivo, passando a fazer mudanças nos seus hábitos e cotidianos.

As práticas diárias que demandaram um menor rearranjo das atividades rotineiras foram as mais incluídas no dia a dia dos participantes, e as práticas que requeriam mais tempo para sua execução não foram cumpridas regularmente pela maior parte dos estudantes. Essas dificuldades vão ao encontro de achados de outros estudos ${ }^{47-50}$, que identificaram a falta de tempo como característica muito prevalente entre estudantes de Medicina brasileiros comparados a estudantes de outros países, sendo a carga horária curricular uma queixa recorrente durante os grupos focais deste estudo.

Eu incorporei na rotina prestar atenção no que eu vejo, para fazer as coisas. Com o tempo, percebi como é bom aproveitar o dia... Há tantas coisas legais que acontecem [durante o dia] e continuamos sem notar. Mesmo que esteja indo para aula ou voltando para casa ... Várias coisinhas do dia. [O programa de meditação] fez muita diferença, descobri um novo prazer: viver o dia prestando atenção no que está acontecendo. Para mim foi muito legal (G6).

Aproximadamente metade dos estudantes que iniciaram o curso participaram de cinco ou mais sessões. Outros estudos com populações semelhantes mostram uma taxa de assiduidade mais alta ${ }^{51,52}$. Entretanto, no nosso caso, foram comuns relatos sobre como os compromissos acadêmicos inviabilizaram a continuidade da prática.

\section{CONCLUSÕES}

Os dados sugerem que o programa desenvolvido foi eficaz em promover as reflexões que tornam intervenções baseadas em mindfulness instrumentos úteis para a promoção de saúde mental. Verificaram-se ampliação do autoconhecimento, redução de sentimentos negativos-pensamentos ruminativos e estabelecimento de rede de apoio entre os próprios estudantes durante e após a aplicação do programa. Entretanto, os resultados devem ser interpretados com cautela, uma vez que novas pesquisas com um maior número de participantes, com metodologias complementares a esta e com maior tempo de seguimento seriam úteis para avaliar melhor os potenciais benefícios da prática na população estudada.

A significativa melhora nos parâmetros analisados corrobora a aplicação da intervenção de meditação como uma prática integrativa e complementar útil no estabelecimento de uma rede de apoio para estudantes de Medicina. Na perspectiva de uma abordagem em grupo, além dos efeitos positivos, a prática se mostrou barata, acessível e de fácil reprodução. Por se 
tratar de uma prática com diversos protocolos estabelecidos e bem estudados, poderia se tornar uma inestimável ferramenta de apoio à saúde mental dos estudantes.

\section{CONTRIBUIÇÃO DOS AUTORES}

Deivisson Vianna Dantas dos Santos, Sabrina Stefanello Heron Eduardo Fabrizzi e Laura Leísa Silveira participaram ativamente da concepção e do delineamento do trabalho, da convocação de participantes, da discussão dos resultados, da redação do manuscrito, da revisão crítica e da aprovação da versão final. Heron Eduardo Fabrizzi e Laura Leísa Silveira foram os condutores das sessões da intervenção. Henrique Cesar Correa Hamilko participou ativamente da discussão dos resultados, da redação do manuscrito, da revisão crítica e da aprovação da versão final.

\section{CONFLITO DE INTERESSES}

Os autores declaram não haver conflito de interesses neste estudo.

\section{FINANCIAMENTO}

Declaramos que não houve financiamento para a realização desta pesquisa.

\section{REFERÊNCIAS}

1. Yusoff MS, Rahim AF, Baba AA, Ismail SB, Pa MN. Prevalence and associated factors of stress, anxiety and depression among prospective medical students. Asian J Psychiatr. 2013 Apr 1;6(2):128-33.

2. Rotenstein LS, Ramos MA, Torre M, Segal JB, Peluso MJ, Guille C, et al. Prevalence of depression, depressive symptoms, and suicidal ideation among medical students: a systematic review and meta-analysis. JAMA. 2016 Dec 6;316(21):2214-36.

3. da Nóbrega S, Moreira T, Dutra EM. Humanização e formação médica: cuidando da saúde mental do estudante de Medicina. Blucher Medical Proceedings. 2014;1(2):94.

4. Stewart SM, Lam TH, Betson CL, Wong CM, Wong AM. A prospective analysis of stress and academic performance in the first two years of medical school. Med Educ. 1999 Apr;33(4):243-250.

5. Hojat M, Robeson M, Damjanov I, Veloski JJ, Glaser K, Gonnella JS. Students' psychosocial characteristics as predictors of academic performance in medical school. Acad Med. 1993 Aug;68(8):635-7.

6. Neumann M, Edelhäuser F, Tauschel D, Fischer MR, Wirtz M, Woopen C, et al. Empathy decline and its reasons: a systematic review of studies with medical students and residents. Acad Med. 2011 Aug 1;86(8):996-1009.

7. Silva FB, Mascia AR, Lucchese AC, De Marco MA, Martins MC, Martins LA. Atitudes frente a fontes de tensão do curso médico: um estudo exploratório com alunos do segundo e do sexto ano. Rev Bras Educ Med. 2009;33(2):230-9.

8. Ramos-Cerqueira AT, Lima MC. La formación de la identidad médica: implicaciones para la enseñanza de graduación en Medicina. Interface Comun Saúde Educ. 2002;6(11):107-16.

9. Dyrbye LN, Thomas MR, Shanafelt TD. Medical student distress: causes, consequences, and proposed solutions. Mayo Clin Proc 2005 Dec 1;80(12):1613-22.

10. Vago DR, David SA. Self-awareness, self-regulation, and self-transcendence (S-ART): a framework for understanding the neurobiological mechanisms of mindfulness. Front Hum Neurosci. 2012 Oct 25;6:296.
11. Kushner K, Marnocha M. Meditation and relaxation. In: O'Donohue $W_{\text {, }}$ Cummings NA, editors. Evidence-based adjunctive treatments. Elsevier; 2011 Apr 28 p. 177-205.

12. Brasil. Portaria n 849 , de 27 de março de 2017. Inclui a Arteterapia, Ayurveda, Biodança, Dança Circular, Meditação, Musicoterapia, Naturopatia, Osteopatia, Quiropraxia, Reflexoterapia, Reiki, Shantala, Terapia Comunitária Integrativa e Yoga à Política Nacional de Práticas Integrativas e Complementares. Diário Oficial da União, Brasília; 2017.

13. Cullen M. Mindfulness-based interventions: an emerging phenomenon. Mindfulness. 2011 Sept 1;2(3):186-93.

14. Vandenberghe $L$, Assunção $A B$. Concepções de mindfulness em Langer e Kabat-Zinn: um encontro da ciência ocidental com a espiritualidade criental. Contextos Clín. 2009;2(2):124-35.

15. Edenfield TM, Saeed SA. An update on mindfulness meditation as a selfhelp treatment for anxiety and depression. Psychol Res Behav Manag. 2012;5:131-141.

16. Khanna S, Greeson JM. A narrative review of yoga and mindfulness as complementary therapies for addiction. Complement Ther Med. 2013 June 1;21(3):244-52.

17. Wanden-Berghe RG, Sanz-Valero J, Wanden-Berghe C. The application of mindfulness to eating disorders treatment: a systematic review. Eat Disord. 2010 Dec 28;19(1):34-48.

18. Ong JC, Ulmer CS, Manber R. Improving sleep with mindfulness and acceptance: a metacognitive model of insomnia. Behav Res Ther. 2012 Nov 1;50(11):651-60.

19. Kabat-Zinn J. Some reflections on the origins of MBSR, skillful means, and the trouble with maps. Contemporary Buddhism. 2011 May 1;12(1):281-306.

20. Shennan C, Payne S, Fenlon D. What is the evidence for the use of mindfulness-based interventions in cancer care? A review. Psychooncology. 2011 July;20(7):681-97.

21. Kabat-Zinn J, Wheeler E, Light T, Skillings A, Scharf MJ, Cropley TG, et al. Influence of a mindfulness meditation-based stress reduction intervention on rates of skin clearing in patients with moderate to severe psoriasis undergoing photo therapy (UVB) and photochemotherapy (PUVA). Psychosom Med. 1998 Sep 1;60(5):625-32.

22. Chiesa A, Serretti A. Mindfulness-based stress reduction for stress management in healthy people: a review and meta-analysis. J Altern Complement Med. 2009 May 1;15(5):593-600.

23. Sauer S, Lynch S, Walach H, Kohls N. Dialectics of mindfulness: implications for western medicine. Philos. Ethics Humanit Med. 2011 Nov;6(1):1-7.

24. Thomas JT, Otis MD. Intrapsychic correlates of professional quality of life: mindfulness, empathy, and emotional separation. J Soc Social Work Res. 2010;1(2):83-98.

25. Mrazek MD, Franklin MS, Phillips DT, Baird B, Schooler JW. Mindfulness training improves working memory capacity and GRE performance while reducing mind wandering. Psychol Sci. 2013 May;24(5):776-81.

26. McConville J, McAleer R, Hahne A. Mindfulness training for health profession students- the effect of mindfulness training on psychological well-being, learning and clinical performance of health professional students: a systematic review of randomized and non-randomized controlled trials. Explore. 2017 Jan 1;13(1):26-45.

27. Gilmartin H, Goyal A, Hamati MC, Mann J, Saint S, Chopra V. Brief mindfulness practices for healthcare providers - a systematic literature review. Am J Med. 2017 Oct 1;130(10):1219-e1.

28. Tripp D. Pesquisa-ação: uma introdução metodológica. Educação e Pesquisa. 2005;31(3):443-66.

29. Turato ER. Métodos qualitativos e quantitativos na área da saúde: definições, diferenças e seus objetos de pesquisa. Rev Saude Publica. 2005;39(3):507-14.

30. Segal Z, Williams J, Teasdale J, Kabat-Zinn J. Mindfulness-based cognitive therapy for depression. 2nd ed. New York: Guilford Publicatons; 2018;44-390.

31. Faria WM, Bógus CM, Mello MD. Grupos focais: experiências precursoras em programas educativos em saúde no Brasil. Bol Oficina Sanit Panam. 1996;120(6):472-482. 
32. Morgan DL. Focus groups as qualitative research. Sage publications; 1996 Oct 23.

33. Wiggins GS. The analysis of focus groups in published research articles. The Canadian Journal of Program Evaluation. 2004 Oct 1;19(2):143-164.

34. Ricoeur P. Entre o tempo vivido e o tempo universal: o tempo histórico. In: Ricoeur P. Tempo e narrativa. Campinas: Papirus; 1997.

35. Ricoeur P. Interpretação e ideologia. 4a ed. Rio de Janeiro: Francisco Alves; 1990.

36. Cormack D, Jones FW, Maltby M. A "collective effort to make yourself feel better": the group process in mindfulness-based interventions. Qual Health Res. 2018 Jan;28(1):3-15.

37. Rodrigues MF, Nardi AE, Levitan M. Mindfulness nos transtornos do humor e ansiedade: uma revisão da literatura. Trends Psychiatry Psychother. 2017 Sept;39(3):207-15.

38. Cairns V, Murray C. How do the features of mindfulness-based cognitive therapy contribute to positive therapeutic change? A meta-synthesis of qualitative studies. Behav Cogn Psychother. 2015 May;43(3):342-59.

39. Fordham B, Griffiths CE, Bundy C. A pilot study examining mindfulnessbased cognitive therapy in psoriasis. Psychol Health Med. 2015 Jan 2;20(1):121-7.

40. Allen M, Bromley A, Kuyken W, Sonnenberg SJ. Participants' experiences of mindfulness-based cognitive therapy: "it changed me in just about every way possible". Behav Cogn Psychother. 2009 July;37(4):413-30.

41. Nolen-Hoeksema S. The other end of the continuum: the costs of rumination. Psychol Inq. 1998 July 1;9(3):216-9.

42. Yalom ID, Leszcz M. The theory and practice of group psychotherapy. 5th ed. New York: Basic Books; 2005.

43. Hjeltnes A, Binder PE, Moltu C, Dundas I. Facing the fear of failure: an explorative qualitative study of client experiences in a mindfulness-based stress reduction program for university students with academic evaluation anxiety. Int J Qual Stud Health Well-being. 2015 Jan 1;10(1):27990.
44. van Aalderen JR, Donders AR, Peffer K, Speckens AE. Long-term outcome of mindfulness-based cognitive therapy in recurrently depressed patients with and without a depressive episode at baseline. Depress Anxiety. 2015 Aug;32(8):563-9.

45. Munshi K, Eisendrath S, Delucchi K. Preliminary long-term follow-up of mindfulness-based cognitive therapy-induced remission of depression. Mindfulness. 2013 Dec 1;4(4):354-61.

46. de Vibe M, Solhaug I, Rosenvinge JH, Tyssen R, Hanley A, Garland E. Sixyear positive effects of a mindfulness-based intervention on mindfulness, coping and well-being in medical and psychology students; Results from a randomized controlled trial. PloS One. 2018;13(4):e0196053.

47. Guillaumie L, Boiral $O$, Champagne J. A mixed-methods systematic review of the effects of mindfulness on nurses. J Adv Nurs. 2017 May;73(5):1017-34.

48. Griffiths K, Camic PM, Hutton JM. Participant experiences of a mindfulnessbased cognitive therapy group for cardiac rehabilitation. J Health Psychol. 2009 July; 14(5):675-81.

49. Meeten F, Whiting S, Williams CM. An exploratory study of group mindfulness-based cognitive therapy for older people with depression. Mindfulness. 2015 June 1;6(3):467-74.

50. Tempski P, Bellodi PL, Paro HB, Enns SC, Martins MA, Schraiber LB. What do medical students think about their quality of life? A qualitative study. BMC Med Educ. 2012 Dec 1;12(1):106.

51. De Vibe M, Solhaug I, Tyssen R, Friborg O, Rosenvinge JH, Sørlie T, et al Mindfulness training for stress management: a randomised controlled study of medical and psychology students. BMC Med Educ. 2013 Dec;13(1):107.

52. Erogul M, Singer G, Mclntyre T, Stefanov DG. Abridged mindfulness intervention to support wellness in first-year medical students. Teach Learn Med. 2014 Oct 2;26(4):350-6. 